\title{
Entropy of the Monomeric Forms of Formic Acid and Acetic Acid
}

\author{
J. O. HALFORD \\ Department of Chemistry, University of Michigan, Ann Arbor, Michigan
}

(Received May 19, 1942)

\begin{abstract}
A recent measurement of the entropy of gaseous formic acid at its equilibrium vapor pressure is combined with vapor density data to give $60.0 \pm 0.3$ as the entropy of the monomer at $25^{\circ}$ and one atmosphere. The corresponding dimer entropy is $83.1 \pm 0.3$ e.u. Pauling's residual entropy of $R \ln 2$ per dimer weight for random orientation of hydrogen bonds in the crystal has been included in the above figures in order to allow a reasonable entropy for the torsional motion of the hydroxyl group in the monomer. The same correction has been applied to a previously published result for the acetic acid monomer to yield $70.1 \pm 1.0$ as a revised value. It is highly probable that only a single significant potential minimum of undetermined breadth and depth occurs in the rotational cycle of the hydroxyl group.
\end{abstract}

\section{FORMIC ACID}

$\mathrm{A}^{\mathrm{c}}$ CCURATE entropy values have recently been obtained by Stout and Fisher ${ }^{1}$ for liquid and gaseous formic acid at $25^{\circ}$ and the equilibrium vapor pressure. Their measurements include the redetermination of the vapor pressure $(43.1 \mathrm{~mm})$ and the heat of vaporization $(4754 \pm 5$ cal. for $46.026 \mathrm{~g}$ ). It will be shown that their results can be combined with Coolidge's ${ }^{2}$ vapor density data to give the entropy of the monomer as a hypothetical perfect gas with a probable error less than 0.3 e.u.

The calculation follows the same lines as the one recently made by the writer for acetic acid. ${ }^{3}$ It is evident from the vapor density measurements, as Coolidge has stated, that at pressures below saturation, in the temperature range from $10^{\circ}$ to $50^{\circ}$, the vapor is adequately described as a mixture of perfect gases in equilibrium according to the equation

$$
(\mathrm{HCOOH})_{2}=2 \mathrm{HCOOH} \text {. }
$$

At higher temperatures and pressures the equilibrium quotient decreases with increasing pressure.

The equation

$$
\log k=24.6070-3754.9 / T-4.7034 \log T
$$

has been derived by the method of zero sums from fifty-four vapor density measurements made at temperatures from $10^{\circ}$ to $80^{\circ}$. It was found that by including some data taken above $50^{\circ}$, the precision in determining the slope in the lower

${ }^{1} \mathrm{~J}$. W. Stout and L. H. Fisher, J. Chem. Phys. 9, 163 (1941).

2 A. S. Coolidge, J. Am. Chem. Soc. 50, 2173 (1928).

3 J. O. Halford, J. Chem. Phys. 9, 859 (1941). temperature range could be increased. This increase occurs at the expense of accuracy in the heat capacity term, which fortunately is of no importance to the present calculation. The method of least squares gives

$$
\log k=27.685-3904.3 / T-5.745 \log T \text {. }
$$

The sum of the squares of deviations, however, is about the same for Eq. (2) as for Eq. (2'). For the heat of dissociation of the dimer, the above equations lead to the expressions

and

$$
\Delta H(\text { dis })=17,182-9.348 T
$$

$$
\Delta H(\text { dis })=17,866-11.399 T
$$

and to the values 14,395 and $14,460 \mathrm{cal}$. at $25^{\circ}$. Further application of the least squares method with $\Delta C p$ arbitrarily set at zero yields $14,272 \mathrm{cal}$, which is below the limit of reasonable interpretation, since the curvature is obvious from the plotted function. By inspection, probable values lie well within the limits $14,400 \pm 120 \mathrm{cal}$.

The probable error of a single determination of $\log k$ may be taken as $\left(\Sigma v^{2} / n\right)^{\frac{1}{2}}=0.0122$, where $v$ is the deviation and $n$ is the number of measurements. This quantity represents the largest reasonable estimate of the probable deviation of the mean from the true value. A more representative estimate would be lower, in the direction of $\left(\Sigma v^{2} / n^{2}\right)^{\frac{1}{2}}$, the quantity used for random deviations in $n$ measurements of a single point. It seems safest here to use the larger probable error. Conversion to a probable error in the heat of dissociation is made by assuming that a plus or minus deviation of 0.0122 in $\log k$ might occur 
with equal probability at either $10^{\circ}$ or $80^{\circ}$, and applying the expression $\Delta H=-R d \ln k / d(1 / T)$. The result is a probable error of $\pm 80 \mathrm{cal}$. In subsequent calculations, the rounded figure $14,400 \pm 100 \mathrm{cal}$. is used for the heat of dissociation.

From Eq. (2), $k_{298.1}=2.361$ and the degree of dissociation $\alpha=(k /(k+4 p))^{\frac{1}{2}}=0.1162$. Individual heats of vaporization for the monomer and dimer may be obtained from the equations

$$
\Delta H_{1}(46 \mathrm{~g})=\frac{\Delta H(\mathrm{vap})}{2}+\frac{1-\alpha}{2} \Delta H(\mathrm{dis})
$$

and

$$
\Delta H_{2}(92 \mathrm{~g})=\Delta H(\mathrm{vap})-\alpha \Delta H(\mathrm{dis})
$$

For the corresponding entropies of vaporization,

$$
\begin{aligned}
\Delta S_{1}(46 \mathrm{~g})=\frac{\Delta H(\mathrm{vap})}{2 T}+\frac{1-\alpha}{2 T} \Delta H(\mathrm{dis}) & \\
& +R \ln \frac{2 \alpha p}{760(1+\alpha)}
\end{aligned}
$$

and

$$
\begin{aligned}
& \Delta S_{2}(92 \mathrm{~g})=\frac{\Delta H(\mathrm{vap})}{T}-\frac{\alpha \Delta H(\text { dis })}{T} \\
&+R \ln \frac{(1-\alpha) p}{760(1+\alpha)},
\end{aligned}
$$

where $\Delta H$ (vap) and $\Delta H$ (dis) apply to $92 \mathrm{~g}$ of the acid and $p$ is the equilibrium vapor pressure.

Numerical substitution into Eqs. (6) and (7) gives 28.48 and 20.11 for the respective entropies of vaporization. By combination with the Stout and Fisher result, 30.82 e.u. per monomer weight of liquid, the entropies of the hypothetical perfect gases at $25^{\circ}$ and one atmosphere are found to be

$$
S_{1}=59.30 \text { and } S_{2}=81.75 \text { (prelim.). }
$$

The sum of probable errors in $S_{1}$ introduced by the heats of vaporization and dissociation and by the pressure and the degree of dissociation is 0.3 e.u., and the combined probable error is 0.2 e.u. The probable error in $S_{2}$ is about the same, in spite of the double weight of acid.

Consideration may now be given to the question of residual entropy in the crystal, as predicted by Pauling. ${ }^{4}$ For this purpose the entropy contributed by torsional motion of the hydroxyl group is found by subtracting the sum calculated for the remaining degrees of freedom from the monomer entropy. All angles between single bonds are assumed to have the tetrahedral

\footnotetext{
${ }^{4}$ L. Pauling, J. Am. Chem. Soc. 57, 2680 (1935).
}

value and interatomic distances are taken as $\mathrm{C}-\mathrm{H}=1.09, \mathrm{C}=\mathrm{O}=1.24, \mathrm{C}-\mathrm{O}=1.43$ and $\mathrm{O}-\mathrm{H}$ $=0.97 \mathrm{~A}$ units. The mean of moment of inertia products for models with cis- and trans-orientation of the hydroxyl group, differing by only 0.1 in their contribution to the entropy, has been used. Frequencies of the normal vibrations are taken from Bonner and Hofstadter and from Herman and Williams ${ }^{5}$ as $3570,2940,1221,1190$, 1111,1075 , and $658 \mathrm{~cm}^{-1}$. There can be little doubt of the correctness of assigning the lowest frequency as a fundamental, and only this one must be accurate and correctly assigned for the present purpose.

The calculated monomer entropy, exclusive of the torsional motion, is 59.25 e.u., to be compared with 59.30 based upon the thermal and vapor density data. The contribution of the internal rotation or vibration is then $0.05 \pm 0.3+S$ (res), where $S$ (res), the entropy at the absolute zero, may be zero, or, if Pauling's prediction is correct, $\frac{1}{2} R \ln 2=0.69$. From these figures the larger torsional entropy, $0.74 \pm 0.3$ e.u., appears by far the more probable. This corresponds to a harmonic frequency range from 380 to $600 \mathrm{~cm}^{-1}$, whereas the zero residual entropy would correspond to the range from 650 to $1150 \mathrm{~cm}^{-1}$, which is less reasonable. The monomer and dimer entropies are therefore revised to

$$
S_{1}=60.0 \pm 0.3 \text { and } S_{2}=83.1 \pm 0.3 .
$$

If the hydroxyl group is arbitrarily treated as a symmetrical rotator with a moment of inertia of $1.35 \times 10^{-40}$ it will contribute in free rotation 4.45 units to the entropy at $25^{\circ}$, leading to a total free rotation entropy of 63.7 . This treatment omits some small subtractions called for by Kassel's determinant. ${ }^{6}$ It is easy to show, however, that the refinements of his interesting and ingenious procedure are numerically unimportant except for molecules of very low molecular weight.

The above calculations indicate that in formic acid an entropy deficiency of $3.7 \pm 0.3$ units results from hindrance of the internal rotation.

\section{ACETIC ACID}

It is probable that acetic acid also has residual entropy at the absolute zero due to random

${ }^{5}$ L. G. Bonner and R. Hofstadter, J. Chem. Phys. 6, 53 (1938); R. C. Herman and V. Williams, J. Chem. Phys. 8, $447(1940)$.

${ }^{8}$ L. S. Kassel, J. Chem. Phys. 4, 276 (1936). 
orientation of hydrogen bonds. The value recently published by the writer should therefore be increased from 69.4 to $70.1( \pm 1.0)$ e.u. The decision is based entirely upon the more accurate formic acid calculation, since an unknown uncertainty in the entropy of liquid acetic acid makes it necessary to leave a relatively wide allowance for error. For this reason the residual entropy problem was not considered in connection with the original acetic acid calculation.

In addition, a revision of the estimated free rotation entropy is in order. The vibrational contribution was taken from the mechanically analogous acetone molecule, for which it had been assumed that rotation of the methyl groups is practically unhindered. A recent simultaneous consideration of acetic acid, acetone and isobutylene, in conference with Dr. R. H. Gillette, has led to the conclusion that the vibrational entropy of acetone and acetic acid should be about 1.2 units higher than originally assigned. This raises the free rotation value for acetic acid from 72.7 to 73.9 , and leaves 3.8 units for the deficiency below the free rotation entropy. Of this quantity, by analogy with acetone, about 0.6 should be subtracted for the methyl group, leaving $3.2 \pm 1.0$ for the hydroxyl group.

\section{THE CARBOXYL GROUP}

The estimated probable errors are large enough to obscure any difference between the entropy deficiencies calculated for the two acids. It is permissible to conclude that the deficiencies are large and roughly equal, and to add the suspicion that a greater hindrance to free rotation may occur in formic acid.

The above revision of the acetic acid entropy produces no appreciable change in the calculated entropy deficiency. Consequently the tentative explanation made in connection with the first calculation is still equally applicable. If hindrance of rotation was ascribed to a single sinusoidal potential valley covering one-third of the cycle, the large deficiency could occur with a potential barrier of approximately 3000 cal. For acetic acid this interpretation could only be called somewhat probable because of the rather large probable error assigned to the entropy deficiency.

The more accurate formic acid result permits a more objective consideration leading to the same type of description. There must be at least one important potential minimum to account for the large deficiency below free rotation $(3.7 \pm 0.3$ e.u.). From the shape of the molecule alone as many as three more minima are possible, but they are readily shown to be relatively unimportant. For this purpose it is convenient to consider the contribution of the torsional motion $(0.75 \pm 0.3$ e.u.) as an entropy of mixing $-R \Sigma X_{i} \ln X_{i}$ where $X_{i}$ is the fraction of molecules to be found in the $i$ th energy state, vibrational or rotational. If, for example, there were two states of approximately equal probability, the entropy would be $R \ln 2$, or 1.38. Still larger values would result from the occurrence of three comparable states, or from the subdivision of either or both of the two states just considered. If all the molecules were divided between two states, about 85 percent of them would be in the more populated one.

It is probable, then, that the one minimum description is at least qualitatively correct, since the occurrence of other important minima would cause distribution of the molecules over two or more comparable states and the resulting entropy would be much larger than the calculated value. The results, however, do not permit any specific conclusions about the height or effective width of the potential valley. The barrier will not be unusually high if the valley does not cover more than about one-third of the cycle.

A single configuration might be considerably stabilized by resonance of the arrangements I and II.
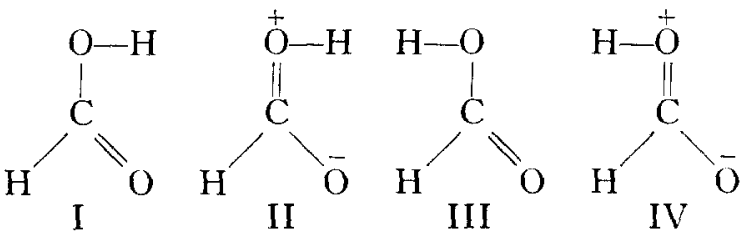

Formulas II and IV would produce potential minima corresponding to cis-trans-isomerism which would presumably favor II because of the attraction of the negatively charged oxygen atom for the proton. If, in addition, a repulsion between the hydrogen atoms occurred, IV would be still less stable relative to II, and the transminimum might be substantially eliminated. The configuration described in terms of the $c i s$-forms I and II would then predominate over the alternative situation embraced by formulas III and IV. 